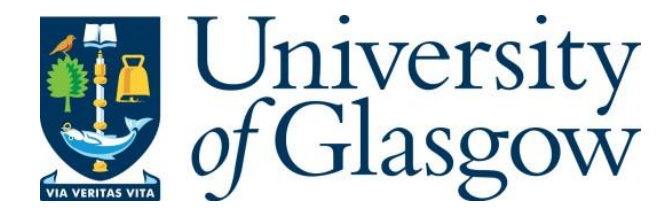

Yalagala, B. P., Deswal, S., Vanjari, S. R. K. and Dahiya, R. (2021) Flexible and Ultrafast Bioresorbable Nanofibers of Silk Fibroin-PVA Composite. In: 2021 IEEE

International Conference on Flexible and Printable Sensors and Systems (FLEPS), 20-23 Jun 2021, ISBN 9781728191737.

There may be differences between this version and the published version. You are advised to consult the publisher's version if you wish to cite from it.

http://eprints.gla.ac.uk/253045/

Deposited on: 7 October 2021

Enlighten - Research publications by members of the University of Glasgow http://eprints.gla.ac.uk 


\title{
Flexible and ultra-fast bioresorbable nanofibers of silk fibroin-PVA composite
}

\author{
Bhavani Prasad Yalagala ${ }^{2}$, Sweety Deswal ${ }^{1}$, Siva Rama Krishna Vanjari ${ }^{2}$, and Ravinder Dahiya ${ }^{1 *}$, Fellow, IEEE, \\ ${ }^{1}$ Bendable Electronics and Sensing Technologies (BEST) Group, University of Glasgow, Glasgow, G12 8QQ, UK \\ ${ }^{2}$ Indian Institute of Technology, Hyderabad, Telangana - 502285, INDIA. \\ *Correspondence to: Ravinder.Dahiya@glasgow.ac.uk
}

\begin{abstract}
Electronic devices capable of easily degradable, physically destructible, and bioresorbable which are prepared using abundant and natural biomaterials has been an interesting research topic. These transient electronic devices help in the reduction of non-degradable electronic waste and can be explored for the next generation intelligent security applications. Here, in the current work a fully transient novel SF-PVA composite based flexible and biocompatible nanofibers were successfully prepared via simple and facile electrospinning based approach. Combining the silk fibroin with PVA has induced different structural transitions i.e. percentage change in the secondary structures which are studied using XRD and FTIR analysis. Besides, the chemical and morphological studies were done using the Raman, and FESEM analysis. The dissolution tests were performed on ultra-thin electrospun nanofibrous mats which revealed the ultra-fast dissolution of nanofibers in both DI water as well as in PBS with a dissolution time of less than 3 seconds. The as prepared nanofibers exhibit programmable dissolutions, superior mechanical flexibility for the different concentration ratio of PVA without further affecting its morphological properties. The tunable degradation rates are primarily due to the change in the structural transitions induced due to the variation in the percentage of the beta sheets which are conformed from the XRD and FTIR-FSD analysis. Thus, the novel nanocomposite biomaterial based nanofibers paves the path towards the development of the upcoming flexible and transient electronic applications.
\end{abstract}

Keywords- Bioresorbable, biomaterial, silk fibroin, biocompatible, nanofiber, electrospinning, dissolution, transient.

\section{INTRODUCTION}

The demand for biocompatible, biodegradable and bioresorbable materials are tremendously increasing due to the increase of e-waste owing to the shorter life time of modern day electronic devices [1-3]. Currently, most of the traditional electronic devices are made with materials which are non-decomposable, non-biocompatible and toxic inorganic materials as well, leading to severe ecological imbalance worldwide. For instance, with the rapid increase of mobile phones, the demand for the materials like indium which is most commonly used materials in displays has been drastically increased. Moreover, the traditional fabrication methodologies like sputtering, e-beam evaporation, etc. are not only complex and need high vacuum but also are costly and energy inefficient [4]. To overcome aforementioned issues, the ultimate solution lies in development of electronic devices with novel green materials which can easily be degraded and finally disappeared after certain time, while using non-conventional fabrication methods. Currently, various materials such as $\mathrm{MgO}, \mathrm{ZnO}$, egg albumin, polyvinylalcohol (PVA), Polyvinylpyrrolidone (PVP) etc. are explored for wide variety of implantable biomedical devices, secure electronic devices such as sensors, memories [5]. This new branch of electronics which uses degradable materials namely transient electronics serves as a best alternative to eradicate the e-waste. Moreover, it provides the electronic devices which can be applied in various security applications such as military applications.

Silk fibroin offers numerous advantages in terms of abundance, superior optical transparency, excellent mechanical flexibility, biocompatibility, and is thus explored as both active and passive material for various flexible electronic applications such as memories [6], sensors [7], nanogenerators [8]. Silk is a natural protein-based polymer exhibit irregular structures with beta-sheets, alpha helices, random coils, turns. Besides, their structural properties can be easily tuned by the addition of various natural and synthetic polymers along with various external treatments like water vapor annealing, thermal pressing, etc. [9-10]. More importantly, the controlled alterations in the molecular conformations of silk fibroin result in fine-tuning various physical and chemical properties. Silk fibroin exhibits two types of molecular conformations of the secondary structures, Silk-I and Silk-II, which are metastable and stable forms. SilkI is a non-crystalline form with more random coils, alpha helices, and side chains and easily soluble in water [11].

On the other hand, Silk-II typically exhibits organized and crystalline structures with more beta-sheets with hydrophobic nature and insoluble in water. Due to their excellent features such as high porosity, excellent mechanical strength, larger surface area to volume ratio, and ease of preparation, Nanofibers were widely explored for numerous applications such as wound dressing, energy storage, transistors, sensors, drug delivery, filtration [12]. Low-cost solution processability makes them the best suitable approach to prepare different composite materials that exhibit different versatile physical, chemical, and mechanical properties, which are very useful for most of the next-generation flexible electronic applications. Generally, most of the traditional nanofibers were prepared using conventional synthetic polymers like PVA, PVP, polylactic acid, polyethylene oxide (PEO), etc., via the facile and straightforward electrospinning-based approach [13]. However, the nanofibers prepared using protein-based silk fibroin are scarce and are least explored for flexible and bioresorbable electronic applications..

\section{EXPERIMENTAL SECTION}

\section{A. Materials and Methods}

The non-dyed Bombyx mori silk yarns were purchased from the M/S Bombyx mori silk yarns and textiles, J\&K, India. The concentrated formic acid (FA) (98\%) was purchased from Rankem chemicals, and the PVA of medium Mw (40,000-50,000) was purchased from Sigma Aldrich and was used in the powder form. Calcium chloride dehydrate ( $\mathrm{CaCl} 22 \mathrm{H} 2 \mathrm{O})$ was purchased from Merck, Germany. A highly purified deionized water (DI) of $18.2 \mathrm{M} \Omega \mathrm{cm}-1$ was used throughout the experiments. X-Ray Diffraction (XRD) studies on silk and SF-PVA were performed using Rigaku Ultima IV diffractometer, and Fourier Transform Infrared (FTIR) spectroscopy studies were performed using the Bruker 
Alpha-II FTIR instrument. The Morphological studies were performed using a sophisticated and powerful field-emission JEOL JEM2100 microscope.

\section{B. Preparation of silk fibroin-PVA solution}

Initially, silk fibroin was prepared using a similar method from our previous work [14]. Briefly, 10 gm of silk yarns were cut into different uniform pieces of length $5 \mathrm{~cm}$ and degummed in $2000 \mathrm{ml}$ of boiled DI water with $0.05 \mathrm{M}$ $\mathrm{Na} 2 \mathrm{CO} 3$ for 30 minutes. Next, the degummed silk fibroin is squeezed and dried overnight in the exhaust and further used to prepare regenerated silk fibroin solution (RSF). An RSF solution was prepared by dissolving the degummed silk fibroin in a solution containing $\mathrm{FA}$ to $\mathrm{CaCl} 2$ with a weight ratio of 19:1. Later, the RSF films were prepared by casting the RSF solution in a petri dish followed by soaking in DI water for 10 hours and removing $\mathrm{CaCl} 2$ by repetitive cleaning. Finally, the silk fibroin solution of the desired concentration is prepared by dissolving in FA and purified using the centrifugation process. The silk fibroin-PVA (SF-PVA) was prepared using the physical mixing of different PVA concentrations varying from $1 \mathrm{mg} / \mathrm{mL}$ to $1.5 \mathrm{mg} / \mathrm{mL}$.

\section{Electrospinning of SF-PVA nanofibers}

he SF-PVA nanofibers were prepared using an optimized concentration of $10 \mathrm{wt} . \%$ SF-PVA solution. The purified and centrifuged solution of $3 \mathrm{~mL}$ was loaded into a $5 \mathrm{~mL}$ syringe with a 26-gauze metallic needle. The electrospinning was performed at an electric field of $1.53 \mathrm{kV} / \mathrm{cm}$ and a constant flow rate of $0.5 \mathrm{ml} /$ hour with the distance between the metallic tip and aluminium foil coated collector as $13 \mathrm{~cm}$. Finally, the thick layered electrospunned nanofibers were collected on an aluminium foil and later used for material characterizations.

\section{RESULTS AND DISCUSSION}

\section{A. Material characteization studies}

To study the crystallinity nature, XRD characterization studies were performed on the as-prepared SF-PVA nanofibers. Fig. 1a shows the XRD spectrum with diffraction peaks located at $2 \theta$ values of $19.27 \mathrm{o}$ and 24.50 corresponding to the Silk-II and Silk-I structure, respectively, consistent with the literature [15]. The results reveal that the prepared nanofibers have a mixed structural composition with both amorphous and crystalline domains. To further understand the material's dominant nature, either crystalline or amorphous, a detailed quantitative analysis was performed on the XRD diffractograms of nanofibers using the XRD deconvolution. The percentage of crystallinity is calculated by the ratio of relative areas under Silk-II to the total area using the formula given in equation (1)

$$
\% \text { Crystallinity }=\left(\mathrm{A}_{\beta} / \mathrm{A}_{\mathrm{T}}\right) \times 100
$$

where $A_{T}$ is the total area (amorphous and crystalline ) and $A_{\beta}$ is the area of crystallinity peaks under Silk-II and Silk-I.

The calculated percentage of crystallinity (beta sheets and beta turns) is approximately $29.78 \%$, significantly less than the amorphous structures. The obtained results suggest that the as-prepared SF-PVA-based nanofibers are very much amorphous and have better flexibility. To further examine the conformational changes in the secondary structures, FTIR spectroscopy was done on the SF-PVA composite-based nanofiber, represented in Fig $1 \mathrm{~b}$. The prominent peaks at
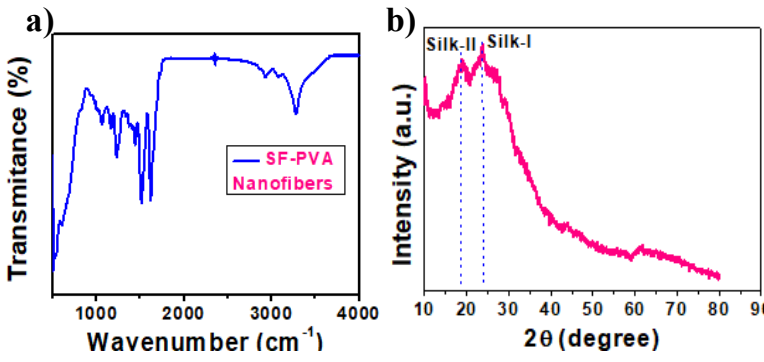

Fig. 1: (a) XRD plot showing both Silk-I and Silk-II structures (b) FTIR plot of SF-PVA.

wavenumbers $1227 \mathrm{~cm}^{-1}, 1509 \mathrm{~cm}^{-1}, 1620 \mathrm{~cm}^{-1}$ clearly confirms silk fibroin's presence with more alpha-helices than beta sheets comparable with the previous studies [16]. Moreover, it is expected that the addition of amorphous PVA to the pristine silk fibroin created several alterations in the secondary structure in the nanofibers might be due to the large amorphous domains of PVA. To better understand the percentage of secondary structures, a detailed Fourier Self Deconvolution (FSD) analysis was performed on both silk fibroin and SF-PVA nanofibers similar to our previous work [14] which is shown in table 1 . The results unveil that the percentage of alpha-helices in the SF-PVA nanofibers is more than the silk fibroin, which coincides well with XRD analysis.

\begin{tabular}{|c|c|c|}
\hline Material & $\begin{array}{c}\text { Alpha } \\
\text { helices (\%) }\end{array}$ & $\begin{array}{c}\text { Other secondary } \\
\text { structure (\%) }\end{array}$ \\
\hline Pure Silk & 23 & 77 \\
\hline Silk-PVA & 32 & 68 \\
\hline
\end{tabular}

Table 1: Percentage alpha sheets calculated from the FSD analysis using Amide-I region of FTIR spectrum.

Fig. 2 shows the Raman spectra of the SF-PVA composite, and the former active band primarily confirms the presence of random coils while the latter two active bands indicate the presence of the crystalline beta-sheets in the SF-PVA composite. The remaining active bands at wavenumbers 885 $\mathrm{cm}-1,1412 \mathrm{~cm}-1,1547 \mathrm{~cm}-1$ confirms the presence of PVA, which matches well with the literature [17].

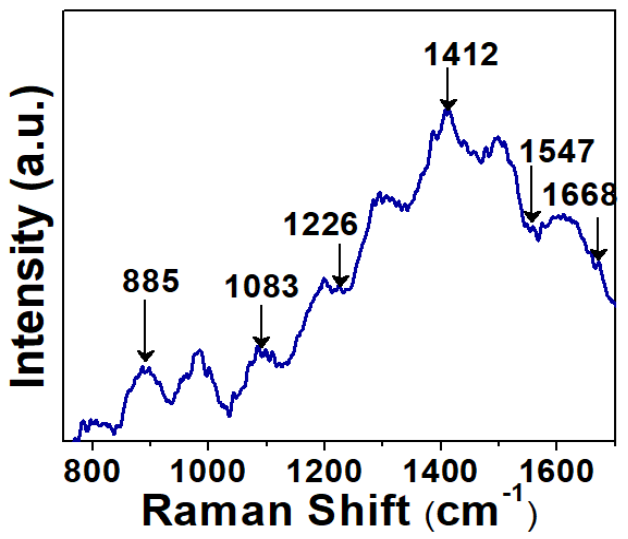

Fig. 2: Raman spectrum of Silk-PVA composite

The morphological studies were performed on both the asprepared pure silk and SF-PVA composite which is clearly shown in Fig 3. Fig. $3 a$ and $3 b$ shows the low and high magnification images of pristine silk with very uniform and highly porous nanofiber mats with no droplets or beads they were formed during electrospinning with an average diameter 


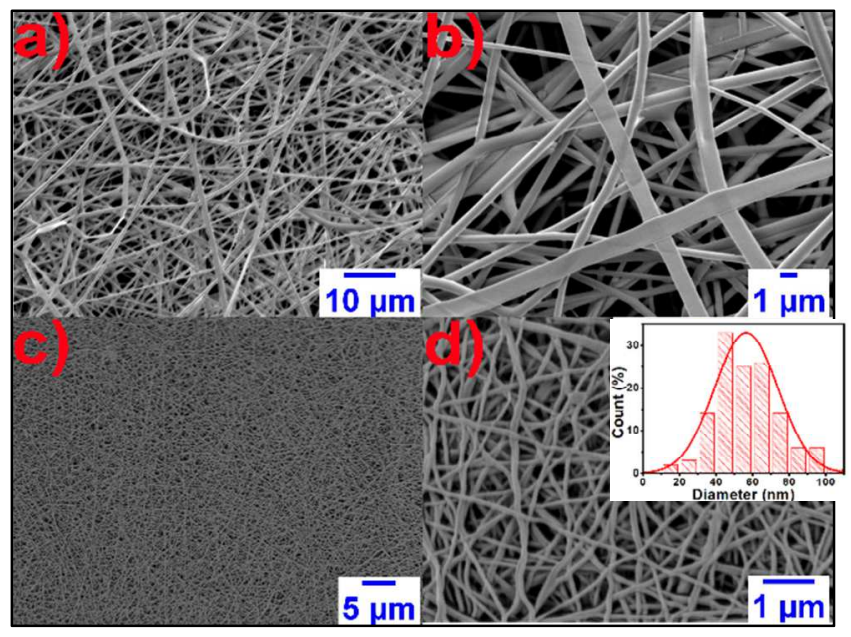

Fig. 3. FESEM images (a) low magnification and (b) high magnification of pristine silk fibroin (c) and (d) low and high magnification images of SFPVA with inset showing the histogram plot showing the diameter of the nanowire of SF-PVA

of $60 \mathrm{~nm}$ approximately. The low and high magnification images of the SF-PVA composite-based nanofibers are illustrated in Fig. 3c and 3d, which are very smooth, uniform, and highly porous similar to that of the pure silk. The SF-PVA nanofiber's average diameter was obtained as $75 \mathrm{~nm}$, calculated using image $\mathrm{J}$ software. The inset of Fig. 3d histogram plot for the SF-PVA nanofibers is depicted the which suggests that the majority of the fibers have a diameter ranging from $40-60 \mathrm{~nm}$.

\section{B. Transient Studies}

The solubility tests were performed on the pristine silk fibroin and different SF-PVA concentrations, shown in Fig. 4. Initially, the pure silk fibroin-based thin films and nanofibers were prepared using the $\mathrm{CaCl} 2-\mathrm{FA}$ based approach and were later used for the dissolution tests. From Fig. 4a, it was observed that the as-prepared nanofibers are insoluble in water due to the higher concentration of hydrophobic domains of amino acids. Fig. $4 \mathrm{~b}$ shows the very smooth, lightweight, and mechanically flexible nanofiber-based film uniformly cut into a square shape of approximately $2 \mathrm{~cm} \mathrm{X} 2$ $\mathrm{cm}$ size before immersion in DI water. Further, the dissolution tests were performed on the SF-PVA composites of different concentrations, i.e., $1 \mathrm{mg} / \mathrm{mL}, 1.5 \mathrm{mg} / \mathrm{mL}, 2$ $\mathrm{mg} / \mathrm{mL}$ indicated as SF-PVA1, SF-PVA1.5, SF-PVA2, which are shown in Fig. $4 \mathrm{c}, 4 \mathrm{~d}$ and $4 \mathrm{e}$, respectively. Interestingly, all the composites dissolve quickly in both DI water and PBS in a short time of 30 seconds, 15 seconds, and 2 seconds, respectively. The as-obtained results suggest that the increase in the concentration of PVA provides faster dissolution rates due to the hydrophilic nature of PVA, resulting in the conversion of the larger number of waterinsoluble hydrophobic to hydrophilic domains inside the SFPVA composites. Moreover, the low molecular weight of PVA further helps in faster dissolution owing to a higher degree of hydrolysis. As a comparison, the dissolution tests were performed on the PVA-based films, which were prepared using the spin coating at a spin speed of $2000 \mathrm{rpm}$ for 30 seconds. The results indicated that the as-prepared PVA-based films were dissolved completely approximately after 5 minutes in DI water and PBS. The thickness of the PVA film is approximately $10 \mu \mathrm{m}$ after spin coating.

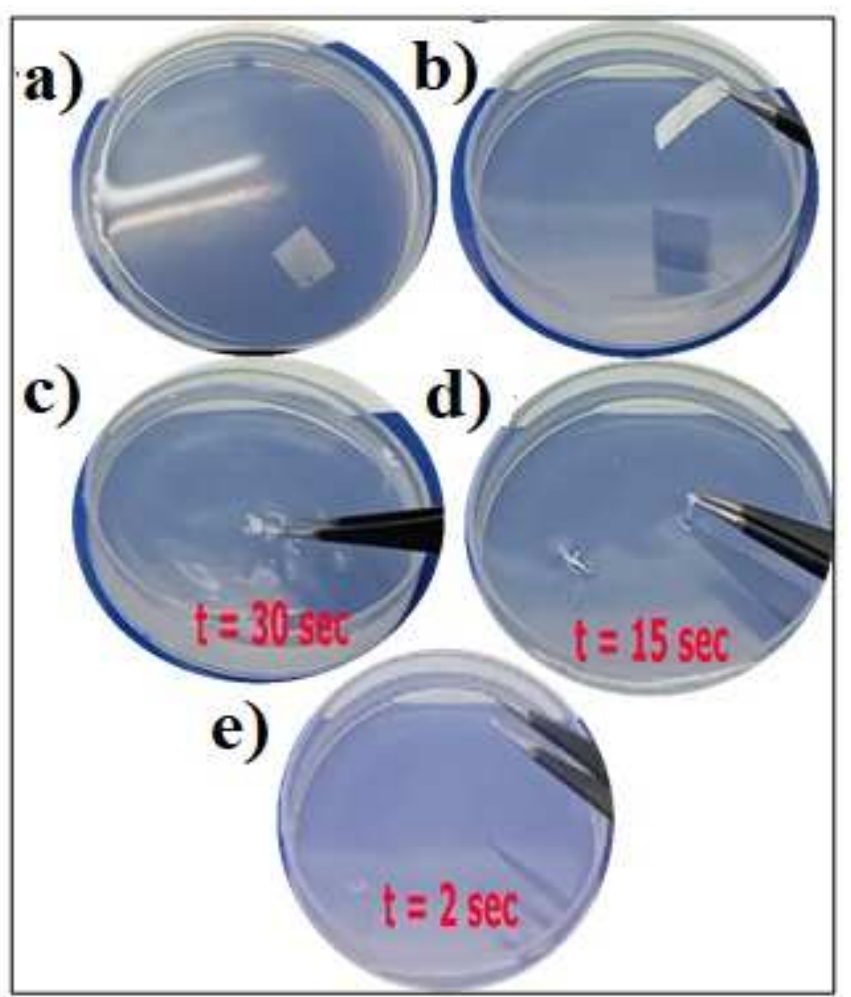

Fig. 4: Dissolution tests with different concentration of PVA in the silk solution (a) pure silk fibroin (b) flexible Silk-PVA film (c) $1 \mathrm{mg} / \mathrm{mL}$ (d) 1.5 $\mathrm{mg} / \mathrm{mL}$ (e) $2 \mathrm{mg} / \mathrm{mL}$ concentration of PVA with different rate of degradations.

\section{CONCLUSION}

In summary, we have successfully prepared uniform, smooth, highly porous, flexible, and lightweight silk-PVA composite-based nanofibers, which can be explored for various electronic applications. Moreover, it was observed that the addition of the increasing concentrations of PVA resulting in the increased number of hydrophilic domains inside the silk Nano fibrils leading to more number alphahelices. Further, to understand the physical transiency property, water dissolution studies were performed on the asprepared silk-PVA films, which reveal that a higher concentration of PVA results in a quicker rate of degradation. Thus, the biocompatible and bioresorbable nanofibers can be used as an active or passive material for the upcoming biomedical and optoelectronic devices and pave the path towards e-waste reduction and future ultra-fast transient electronics.

\section{ACKNOWLEDGEMENT}

This work is supported in part by Engineering and Physical Science Research Council (EPSRC) through Engineering Fellowship (EP/R029644/1) and European Commission through grant references (H2020-MSCAITN2019-861166) 


\section{REFERENCES}

[1] F.Song, H. Wang, J. Sun, B. Dang, H. Gao, M. Yang, and Y. Hao, "Solution-processed physically transient resistive memory based on magnesium oxide". IEEE Electron Device Lett., vol.40, pp 193-195, 2018 .

[2] B. Yalagala, S. Khandelwal, J. Deepika, and S. Badhulika, "Wirelessly destructible MgO-PVP-Graphene composite based flexible transient memristor for security applications". Mater. Sci. Semicond. Process., vol 104, pp 673-680, 2019.

[3] H. Cheng, and V. Vepachedu, "Recent development of transient electronics". Theor. Appl. Mech. Lett., vol 6, pp 21-31, 2016.

[4] B.Yalagala, P. Sahatiya, C.S.R. Kolli, S. Khandelwal, V. Mattela, and $\mathrm{S}$. Badhulika, " $\mathrm{V}_{2} \mathrm{O}_{5}$ nanosheets for flexible memristors and broadband photodetectors" ACS Appl. Nano Mater., vol 2, pp 937-947, 2019.

[5] K.K. Fu, Z. Wang, J. Dai, M. Carter, and L. Hu, "Transient electronics: materials and devices". Chem. mater., vol 28, pp 3527-3539, 2016.

[6] H. Wang, B. Zhu, H. Wang, X. Ma, Y. Hao, and X. Chen," UltraLightweight Resistive Switching Memory Devices Based on Silk Fibroin". Small, vol 12, pp 3360-3365, 2016.

[7] F. He, X. You, H. Gong, Y.Yang, T. Bai, W. Wang, and M. Ye, "Stretchable, biocompatible, and multifunctional silk fibroin-based hydrogels toward wearable strain/pressure sensors and triboelectric nanogenerators". ACS Appl. Mater. Interfaces., vol 12, pp 6442-6450, 2020 .

[8] D.L. Wen, X. Liu, H.T. Deng, D.H. Sun, H.Y. Qian, J. Brugger, and X.s. Zhang, "Printed silk-fibroin-based triboelectric nanogenerators for multi-functional wearable sensing”. Nano Energy, vol 66, pp 104-123, 2019.

[9] W. Hosakun, Y.Hosakun, D. Dudić, V. Djoković, and L. Csóka, "Dependence of mechanical and electrical properties of silver nanocubes impregnated bacterial cellulose-silk fibroin-polyvinyl alcohol films on light exposure". Polym. Test., vol 71, pp 110-114, 2018 .

[10] W. Li, J. Wang, H. Chi, G. Wei, J. Zhang, and L. Dai,” Preparation and antibacterial activity of polyvinyl alcohol/regenerated silk fibroin composite fibers containing Ag nanoparticles". J. Appl. Polym. Sci., vol 123, pp 20-25, 2012

[11] F. Zhang, Q. Lu, J. Ming, H. Dou, Z. Liu, B. Zuo, and X. Zhang, "Silk dissolution and regeneration at the nanofibril scale". Int. J. Mater. Chem. B, vol, 2, pp 3879-3885, 2014.

[12] .Xue, T. Wu, Y. Dai, \& Y. Xia, "Electrospinning and electrospun nanofibers: Methods, materials, and applications". Chem. Rev., vol 119, pp 5298-5415, 2019.

[13] H.M. Ibrahim, and A. Klingner. "A review on electrospun polymeric nanofibers: Production parameters and potential applications". Polym. Test, vol 90, pp 647-653, 2020

[14] D.S.K. Gunapu, Y.B. Prasad, V.S. Mudigunda, P. Yasam, A. .K. Rengan, R. Korla, and S.R.K. Vanjari, "Development of robust, ultrasmooth, flexible and transparent regenerated silk composite films for bio-integrated electronic device applications". Int. J. Biol. Macromol., vol 176, pp 498-509, 2021.

[15] J. Kundu, Y.I. Chung, Y.H. Kim, G. Tae, and S.C. Kundu, "Silk fibroin nanoparticles for cellular uptake and control release". Int. J. Pharm. vol 388, pp 242-250, 2010.

[16] M. Dodel, N.H. Nejad, S.H. Bahrami, M. Soleimani, and H. HanaeeAhvaz, "Modifying the mechanical properties of silk nanofiber scaffold by knitted orientation for regenerative medicine applications". Cell. Mol. Biol., vol 62, pp 16-25, 2016.

[17] B.N. Singh, and K. Pramanik, "Development of novel silk fibroin/polyvinyl alcohol/sol-gel bioactive glass composite matrix by modified layer by layer electrospinning method for bone tissue construct generation". Biofabrication, vol 9, pp 15-28, 2017. 